\title{
Humanistic Management - Sucks Less and Better for your Health
}

\author{
Michael Pirson ${ }^{1}$
}

Published online: 29 June 2018

(C) Springer International Publishing AG, part of Springer Nature 2018

How are you doing?

How well did you sleep last night?

Asking about your well-being and the quality of your sleep may reveal important insights for good organizing practices. If these questions are asked with authentic curiosity the resulting insights could transform businesses. That is at least what more and more CEOs are finding out.

Not so long ago, Arianna Huffington, co-founder and editor in Chief of the Huffington Post, had a breakdown caused by sleep deprivation. Since then she has become an advocate of the importance of sleep. She argues that "we are in the midst of a sleep deprivation crisis, with profound consequences to our health, our job performance, our relationships and our happiness. What we need is nothing short of a sleep revolution: only by renewing our relationship with sleep can we take back control of our lives."1

There is no lack of good sleep advice, still business culture seems to celebrate those that can operate tirelessly on four hours of sleep (and apparently such people exist).

\section{Investors Causing Restless Nights}

Enter Larry Fink, CEO of Blackrock, one of the world's largest asset management companies. Fink commands close to $\$ 7$ TRILLION USD under management. His company owns 3-7\% of assets of most, if not every, publicly listed company in the United States. Fink's recent letter to CEOs reportedly caused sleepless nights. At the World Economic Forum in Davos and beyond, Fink's letter became the conversation item; a signal that it jolted business leadership awake. (Check out the letter here: https:/www.blackrock.com/corporate/investorrelations/larry-fink-ceo-letter).

${ }^{1}$ http://ariannahuffington.com/books/the-sleep-revolution-tr/the-sleep-revolution-hc

Michael Pirson

pirson@fordham.edu

1 International Humanistic Management Association, Fordham University, Bronx, NY, USA 
If you read the letter it is surprising that it would cause much fuss at all. Fink is asking CEOs to articulate more clearly what their social purpose is and adapt their corporate governance systems to ensure long-term value creation. Why should this be a problem?

I have talked a lot with my students and executives about the letter. They generally conclude that both social legitimacy and long-term oriented corporate governance are at odds with current business culture. Business culture and business practice are widely thought of only in terms of short-term oriented profit maximization. People hate business because it neglects concerns for human dignity and well-being. R. Edward Freeman (in this issue) calls this the "business sucks" narrative.

In his essay, Freeman (2018) suggests that the "business sucks" story is one of the dominant modes of thought in our society. It is indicative of a profound mistrust and misunderstanding of the role of business. Freeman suggests that there reigns a dominant myth in society that business occupies the moral low ground, separate from ethics or a humanistic concern. How could one ever envision business having a social purpose in that case? Freeman's essay shows how the enactment of this story underlies business thinking among many managers and business theorists (including those who have trouble with Fink's letter). The essay concludes with a suggestion to rethink and reinvent this story along more realistic and more empowering lines. Freeman suggests that a humanistic, values-based form of capitalism, or stakeholder capitalism, offers a better way to understand the complex role that business does and should play in modern society.

In the light of this essay, one could interpret Fink's letter as a fundamental challenge to corporate executives to ditch the "business sucks" narrative. This is indeed a monumental task which can keep you up at night. To highlight how widespread and deeply rooted this story is, let's consider the case of Kevin $\mathrm{O}^{\prime}$ Marah, a business insider and columnist at Forbes, the business magazine. He writes: "I hate business but I love Larry Fink:"

"Business, Especially In Big, Public Companies Is All About Money. Executives Have Only One Real Duty: To Maximize Shareholder Value. Lip Service About Other Accountabilities Notwithstanding, The Fact Is That C-Level Executives' Responsibility Forces Them To Choose The Path Of Highest Risk-Adjusted Net Present Value.

This drives all kinds of objectionable behavior from exploitative labor practices and pollution to tax evasion. Individual executives may hate it as a matter of conscience, but they are expected to suck it up and, if necessary, do the dirty work.

Equity markets are soulless by design, with a harsh binary logic that recognizes only two signals: buy or sell. Harnessed, as it is now, to the frenetic news cycle of our digital age this engine has become increasingly ill-suited to long-term thinking. At the limit we are tempting revolution by maniacally chasing profit at all cost. Politics reflects this dissatisfaction and is issuing a warning we'd be wise to heed.

It makes me fear for the future." 2

O'Marah has high hopes for Larry Fink because he is asking business to suck less. And that may scare CEOs who have spent their lifetime defending current practices. For many other people including social activists, Larry Fink's letter is a trivial statement, which only matters

${ }^{2}$ https://www.forbes.com/sites/kevinomarah/2018/01/22/i-hate-business-but-i-love-larry-fink/\#4f67b9d87833 
because of who is saying it. Much like $\mathrm{O}^{\prime}$ Marah they hope that Fink' exhortation can lead to a better system that increases the well-being of all. ${ }^{3}$

\section{Purpose, Sleep and Well-Being}

To many enlightened business leaders, social purpose is seen as giving clarity and reducing complexity. It helps management run a business better because the social purpose can guide decision making at many levels. Having a clear social purpose allows one to engage important stakeholders and creates a collaborative advantage. For the CEO, social purpose helps decrease cognitive overload in the brain and therefore improves sleep.

That insight we label humanistic management. It roughly states that ethics and purpose can be much better strategy and is ultimately better for everyone's well-being (Dierksmeier 2016; Dierksmeier et al. 2011; Mele 2003, 2009; Pirson 2017b). Empirical evidence highlights that people who sleep better are also making better decisions and are therefore better managers. In turn, increasing evidence shows that sleep quality is affected by how much people feel their life is meaningful and has purpose. A recent study raises the possibility that sleep could be affected by the degree to which someone feels like his or her life is purposeful or meaningful. Arlener Turner, Christine Smith and Jason Ong of the Northwestern University School of Medicine found that people who reported having a greater sense of purpose in life also reported getting better sleepeven when taking into consideration age, gender, race and level of education. ${ }^{4}$

Humanistic management is the label we use to describe organizing practices that allow people to envision a world where business sucks less and managers can sleep better (and for that matter everyone around them sleeps better too). Humanistic management positions organizational activity around the protection of dignity and the promotion of well-being (Pirson 2017b, c). We have long argued this is better business. It is also very much in line with what Blackrock describes as its guiding principles:

"We believe that companies with sound corporate governance practices, including how they manage the environmental and social aspects of their operations, offer better riskadjusted returns over time."

This thinking is indeed a big challenge for traditional management and is easily dismissed as socialist, interventionist, Marxist etc. We think humanistic management thinking can provide a basis for those managers that sleep fitfully in response to Larry Fink's letter. We further argue that it can provide the foundation of a bolder, more empowering narrative for business and organizing in general, much like Ed Freeman suggests.

The journal has published several pieces that relate to a better narrative (Dion 2017; Lawrence and Pirson 2015; Lovins 2016; Pirson 2016, 2017a, b, c, 2018; Waddock 2016). In a second contribution in this issue, Sandra Waddock (2018) is exploring the possibility of building such a narrative. She is exploring core memes and metrics which she argues need to shift to support a more empowering humanistic narrative for business and beyond. Her conclusions are sobering, however, as the current status of the

\footnotetext{
${ }^{3}$ https://www.cnbc.com/2018/06/12/capitalism-may-need-modernizing-says-billionaire-hedge-fund-managerpaul-tudor-jones.html

${ }^{4}$ https://www.psychologytoday.com/us/blog/the-power-rest/201707/purpose-and-sleep

https://www.scientificamerican.com/article/the-secret-to-a-better-nights-sleep-a-sense-of-purpose/
} 
conversation is very much based on staid memes and outdated categories that are holding people in an intellectual cage. There seems to be a lack of coherent memes used by those entities that address many of the concerns related to dignity violations and well-being promotion. Waddock is concluding her paper suggesting there is much opportunity to seed new memes and metrics and do so in a concerted manner.

The editorial team of the Humanistic Management Journal consider dignity and wellbeing central memes of a more life-conducive economic system (Mele 2003, 2009; Pirson 2016; Pirson et al. 2016). We encourage everyone else so inclined to promote this movement toward more humanistic management practices to enhance human flourishing. Wouldn't you rather dream of business acting in this manner than endure the nightmare of the business sucks narrative?

\section{Further Memes: Mindfulness, Resilience, and Global Health}

Some other memes that are catching on relate to mindfulness and compassion. In fact, one CEO in the fashion industry pointed to the need for more mindfulness dealing with Larry Fink's letter; a practice she had adopted long ago to help her sleep better. In a third contribution, Kevin Jackson (2018) is exploring these increasingly popular memes and their potential contribution to better ethical business practice.

Jackson's article aims to provide a standpoint from which to critically address two broad concerns. The first concern surrounds a naïve view of mindfulness, which takes it as a given that it is a good thing to cultivate mindfulness and attendant qualities like compassion because these virtues are key to improving the quality of life and bettering effective decision-making within business. The second concern is that Western virtuebased business ethics is largely confined to academic philosophical theories. As such, virtue-driven business ethics is often more centered around developing theoretical wisdom than developing "hard core" practical wisdom earned through yoga asanas, meditation, chanting, and breathing, whereas for contemplative practices the reverse is the case, with practical wisdom ("knowing how-to") emphasized over theoretical wisdom ("knowing that").

Jackson's article examines prospects for cross-fertilization between, on the one hand, mindfulness and compassion interpreted as virtues in Eastern contemplative practices, and on the other hand, mindfulness and compassion as interpreted within Western virtue-oriented business ethics. The first theme is that mindfulness and compassion represent key virtues within contemplative practices. This indicates a promising touchpoint between Eastern and Western traditions: their respective focus upon character, inner states, intrinsic motivation, and self-improvement toward ethicality in the world. The second theme is that such virtues in Eastern contemplative practices, as well as character traits integral to Western virtue-oriented approaches, denote contested "normative-interpretive" concepts that engage philosophical debate rather than indisputable empirical-criterial concepts that can be taken at face-value. The third theme advocates moving beyond behaviorist and neuropsychological accounts of virtue, approaching character traits of Eastern contemplative practice and Western virtue ethics through nonscientific inquiry into normative interpretive questions concerning such virtues (questions about meaning, responsibility, the nature of the self, reasons for acting). 
If mindfulness and compassion are critically relevant to better/more ethical business practice, then the questions are 1) how can we teach mindfulness and 2) how can we teach management more mindfully. In the fourth contribution, Benito Teehankee is answering the latter question. In his article on humanistic management pedagogy, Teehankee (2018) is proposing Critical Realist Action research as a form of mindful management education. He reports on teaching experiences accumulated over the past 6 years at a leading business school in the Philippines. The introduction of action research, which is based on critical realist philosophy of science, was intended to enable the university's MBA graduates to become reflexive and humanistic agents of change in their work contexts through the application of observation, critical reflection, collaborative analysis and action and scholarly skills. He reports that the implementation of the action research requirement is beginning to yield positive results based on the types of projects implemented by the students and their resulting sense of efficacy in the workplace. Challenges in implementation include the need to further hone student skills in pursuing more emancipatory projects and the need to further orient faculty in the critical realist philosophy underlying action research, as distinct from traditional positivism.

Connecting the role of mindfulness with organizational health, Shana Hormann (2018) is exploring resilience as a basis for organizational success. Addressing Larry Fink's challenge that CEO's manage risk better, resilience can be key to handle these future challenges. In a fifth contribution aimed at practitioner audiences, she explores the question of what carries people, organizations, and communities through traumatic times? She argues that as a construct, resilience is built on the underlying assumption that an individual or organization has undergone a situation of 'significant adversity' and has adapted positively, returning to or increasing in performance and psychological wellbeing. Resilience is an important quality for leaders who are committed to the sustained health of organizations. Since current and future changes to organizations may be drastic and potentially traumatic, this paper offers recommendations for addressing organizational trauma as well as strengthening resilience, individually and collectively. Hormann shares stories from several organizations, demonstrating the importance of leadership and resilience in the face of trauma.

In a sixth contribution aimed at policy makers, Frederik Ahen is suggesting to connect public policy discourses on ecological governance with those on global health. Ahen (2018) argues that global health and environmental wellbeing are mutually reinforcing and interdependent. This mutuality invokes two major analytical orientations: it emphasizes a direct nexus between ecological strategies and global health outcomes. These in turn revitalize the essential quest for comprehensive policies and responsible strategies for enhancing both ecology and health within the discourse of sustainability. Ahen addresses the question: Why and how might ecological strategies be embedded in corporate day-to-day actions to produce optimal outcomes that have positive effects on global health and human dignity?

Despite the various major impediments, he suggests that there are several grounds for optimism with a move to humanistic eco-centrism via deliberative democratic procedures. His paper provides an interdisciplinary theoretical model that seeks to reorient strategies towards restoration, protection, mitigation, adaptation, harm avoidance and innovative sustainability of the whole economic gamut and biodiversity that supports global health. Thus, Ahen rearticulates ecological sustainability in terms of its most fundamental means and end: sustainable global health and the tutelage of human dignity. 


\section{Introducing a New Section: Future Challenges}

As we have argued before organizational practices will be seen as effective when they are future proof. The ultimate success of the humanistic conception of business and the economy lies not in the past, but in how we position ourselves in the present to meet the needs of the future. As with any management model, the principles behind humanistic management theories have to pass the test of time and must be interpreted continuously in light of new developments.

At present, humanity faces various serious challenges in the natural, social, and digital realm. Problems of sustainability, concerns of inequality, questions of global governance, and the wide field opened up by digitization (blockchain technology, cryptocurrencies, internet of things, artificial intelligence, etc.) must be addressed in order to clarify how the foundational principles of humanistic management can also hold true under the altered circumstances that await us. Consequently, we - the editors - wish to dedicate a new section of the Humanistic Management journal to these novel challenges.

Exploring, however, is different from surveying. Whereas settled affairs lend themselves to comprehensive analysis and roundabout perspectives where, ideally, we find 'all things considered,' the chartering of new territories will of necessity be less certain and complete. For the investigation of the future challenges of humanistic management we consequently decided also for a different format amenable to such investigative intellectual endeavors.

Instead of requiring the traditional length and the conventional features of journal articles (extensive literature review, theory gap definition, clarification of theoretical contribution, etc.), in this section we encourage authors to give us their unfinished (albeit not unpolished) thoughts, to afford us glimpses into 'works in progress' or onto emergent phenomena, to provide us with fresh angles and untested viewpoints. The contributions need not be the final word on the topic at issue but should rather open up new perspectives and stimulate further discussions. In short, the form of the articles submitted for this section can be as exploratory as their content in that, apart from conventionally crafted texts, we also welcome and support 'pro \& con' debates, 'question \& answer' formats as well as narrative and opinion-based pieces.

Starting with this issue we will include several such 'Future Challenge' pieces. One of the most discussed future challenges is that of technology and money. Blockchain technology has an apparent multitude of applications. Bitcoin and other forms of digital currency have occupied the popular press for some time now. It is therefore relevant to sketch out the moral claims for and against the usage of such technology.

Claus Dierksmeier (2018) outlines the normative claims of two different digital money communities: Bitcoin and RIPPLE. He argues that the cryptocurrencies Bitcoin and Ripple stand for two different approaches to the question as to which impact money ought to have in people's lives, and as to how individuals in turn can influence the nature and future of money.

In a response, Peter Seele (2018) is exploring the downsides and moral hazards of such technology. Among the most critical issues are black market transactions of weapons used in terrorist attacks, drugs, or child pornography. Additionally, cryptocurrencies are more and more found in blackmailing people and as payment for ransom-ware and other computer viruses (Wannacry was a remarkable example). Money laundering also is on the rise via cryptocurrencies. Seele argues that the nefarious use of cryptocurrencies threatens the prosocial potential of cryptocurrencies and in general makes criminal activity easier and less likely to track down by legal authorities. He closes by discussing current debates about emerging regulation. 
The Editorial team of the Humanistic Management Journal hopes that you find the current contributions interesting, enlightening and above all not sleep inducing. We also hope that they can contribute to a better night of sleep and increase your well-being.

\section{References}

Ahen, F. 2018. Dystopic prospects of global health and ecological governance: Whither the eco-centrichumanistic CSR of firms? Humanistic Management Journal 3 (1): 105-126.

Dierksmeier, C. 2016. Reframing economic ethics: The philosophical foundations of humanistic management. Heidelberg/New York: Springer.

Dierksmeier, C. 2018. Just HODL? On the moral claims of bitcoin and Ripple users. Humanistic Management Journal 3 (1): 127-131.

Dierksmeier, C., W. Amann, E. Kimakowitz, H. Spitzeck, and M. Pirson, eds. 2011. Humanistic ethics in the age of Globality. London/New York: Palgrave.

Dion, M. 2017. Corporate citizenship, social responsibility, and sustainability reports as "would-be" narratives. Humanistic Management Journal 2 (1). https://doi.org/10.1007/s41463-41017-40022-x.

Freeman, E. 2018. The "business sucks" story. Humanistic Management Journal 3 (1): 9-16.

Hormann, S. 2018. Exploring resilience: In the face of trauma. Humanistic Management Journal 3 (1): 91-104.

Jackson, K. 2018. Interpreting the virtues of mindfulness and compassion: Contemplative practices and virtueoriented business ethics. Humanistic Management Journal 3 (1): 47-69.

Lawrence, P.R., and M. Pirson. 2015. Economistic and humanistic narratives of leadership in the age of globality: Toward a renewed Darwinian theory of leadership. Journal of Business Ethics 128 (2): 383-394.

Lovins, H. 2016. Needed: A better story. Humanistic Management Journal 1 (1): 75-90.

Mele, D. 2003. The challenge of humanistic management. Journal of Business Ethics 44 (1): 77-88.

Mele, D. 2009. Current trends of humanism in business. In Humanism in business: Perspectives on the development of a responsible business society, ed. H. Spitzeck, M. Pirson, W. Amann, S. Khan, and E. von Kimakowitz, 170-184. Cambridge, U.K.: Cambridge University Press.

Pirson, M. 2016. Editorial: Welcome to the humanistic management journal. Humanistic Management Journal 1 (1): 1-7.

Pirson, M. 2017a. Better stories needed: How meaningful narratives can transform the world. Humanistic Management Journal 2 (1): 1-6.

Pirson, M. 2017b. Humanistic management-protecting dignity and promoting well being. Cambridge: Cambridge University Press.

Pirson, M. 2017c. In a time of global upheaval-humanistic management thinking needed more than ever. Humanistic Management Journal 1 (2): 1-3.

Pirson, M. 2018. Reclaiming our humanity-a cornerstone for better management. Humanistic Management Journal 2 (2): 1-5.

Pirson, M., K. Goodpaster, and C. Dierksmeier. 2016. Guest editors' introduction: Human dignity and business. Business Ethics Quarterly 26 (4).

Seele, P. 2018. Let us not forget: Crypto means secret. Cryptocurrencies as enabler of unethical and illegal business and the question of regulation. Humanistic Management Journal 3 (1): 133-139.

Teehankee, B. 2018. Critical realist action research and humanistic management education. Humanistic Management Journal 3 (1): 71-90.

Waddock, S. 2016. Foundational memes for a new narrative about the role of business in society. Humanistic Management Journal 1 (1): 91-105.

Waddock, S. 2018. Narrative, memes, and the prospect of large systems change. Humanistic Management Journal 3 (1): 17-45. 